ISBN 978-981-11-0008-6

Proceedings of 2016 6th International Workshop on Computer Science and Engineering

(WCSE 2016)

Tokyo, Japan, 17-19 June, 2016, pp. 552-558

doi: $10.18178 /$ wcse. 2016.06 .093

\title{
The Management TQF Database Systems
}

\author{
Jeerasak Phumcharoen ${ }^{+}$ \\ Rajamangala University of Technology Suvarnabhumi/Information Technology Department, Suphan Buri, \\ Thailand
}

\begin{abstract}
Thailand Quality Framework (TQF) is mainly the framework that is used to regulate all the higher educations in Thailand. The framework provides basic requirements for all curriculums in higher education in Thailand. Therefore, all the curriculums taught in Thailand must comply with the framework. When the initial version of the framework was introduced to the universities, the framework was completed to deploy to each curriculum, as there are many documents to involve in the process. Therefore, in order to improve the quality assurance of the framework to the faculties, this paper proposed the development of the Management TQF database system (M-TQF). In this work, all of the satisfaction of the actors that interacts with the framework including the lecturer, staff of the Faculty of Science and Technology in the Rajamangala University of Technology Suvarnabhumi were collected. Then the sample was purposively selected from Computer Science and Information Technology programs and analyzed. Finally, based on the information analyzed, the database of the TQF in the Faculty of Science and Technology was proposed to improve the deployment of the new curriculum in the field of Information Technology.
\end{abstract}

Keywords: Thai qualification framework, TQF, management, information technology.

\section{Introduction}

Before the year 2005, all the curriculums in higher education in Thailand were subjectively designed by each university. This causes the problem of quality of the education in higher education in some universities. Therefore, the Office of the Higher Education Commission (OHEC) solved the problem by introducing Thailand Qualification Framework (TQF). TQF is the framework that provides the basic requirements that all the curriculums in Thailand must follow. The framework also provides different requirements from curriculum to curriculum. For the undergraduate program, its core focuses on five areas including ethics, knowledge, intellectual skills, interpersonal and accountability, and a numerical analysis skill, communications and information technology [1]. In addition, TQF also specified additional criteria for distance learning curriculum and graduate degree curriculum [2]. The main goal of the framework is to provide minimum education quality to all of the universities in Thailand and allows comparison of the curriculum quality among the universities in Thailand.

TQF is the process of developing the process of teaching and learning approaches. In the TQF framework, the process starts by planning the teaching and learning processes and performing according to the designed plan. After performing, the goals were evaluated as checkpoints. If some checkpoints failed. The framework allows the process to be adjusted in the next education year. Then the entire process follows the same repeatedly until the curriculum meets the quality standard. However, the process of developing the curriculum is a big process, as it requires a large amount of detailed documents to be written. As the result, the development process of the curriculum becomes redundant and delayed.

Therefore, this work proposed the Management TQF database system (M-TQF) that helps the development process by reducing human errors and redundancy in development curriculum process,

\footnotetext{
+ Corresponding author. Tel.: +6684-1113204.

E-mail address: jeerasak.ph@rmutsb.ac.th.
} 
summarizing the report, and evaluating the curriculum against the TQF. As the result, the system gives the self-assessment report to the user in order to submit this to OHEC.

\section{Literature Review}

In this section, the literature reviews are studied in different fields, which are the concept of the Thailand Qualification Framework, and the existing studies in document management systems field.

\subsection{Thailand qualification framework}

Thailand Qualification Framework (TQF) is one of the country policies to improve the quality of higher education so that the quality of the education can be measurable. The framework also provides different requirements from curriculum to curriculum. For the undergraduate program, its core focuses on five areas including ethics, knowledge, intellectual skills, interpersonal and accountability, and a numerical analysis skill, communications and information technology [1]. In addition, TQF also specified additional criteria for distance learning curriculum and graduate degree curriculum [2]. The main goal of the framework is to provide minimum education quality to all of the universities in Thailand and allows comparison of the curriculum quality among the universities in Thailand. There are seven types of document in the framework, which are TQF1 to TQF7 as shown in Table 1.

Table 1. Document Description of TQF 1-7

\begin{tabular}{|l|l|}
\hline Topics & \multicolumn{1}{c|}{ Description } \\
\hline TQF 1 & Qualification Standards of the faculty/ 4 Majors was already: Nursing, Logistics, Computer, Engineering \\
\hline TQF 2 & Details of the Curriculum. \\
\hline TQF 3 & Course Description \\
\hline TQF 4 & Details of field experiences \\
\hline TQF 5 & The result of the operation of the course. \\
\hline TQF 6 & Report on the implementation of field experiences. \\
\hline TQF 7 & Report on the implementation of the curriculum. \\
\hline
\end{tabular}

First, TQF1 is the framework of the curriculum that specifies the learning outcome of the graduate such that what knowledge the student should know. The purpose of the TQF1 is to allow all the graduates in the same curriculum of the university having the same minimum knowledge. The process of TQF1, as shown in "Fig. 1," creation starts from OHEC issuing the minimum requirements and getting feedback from public hearing until the TQF1 is satisfied.

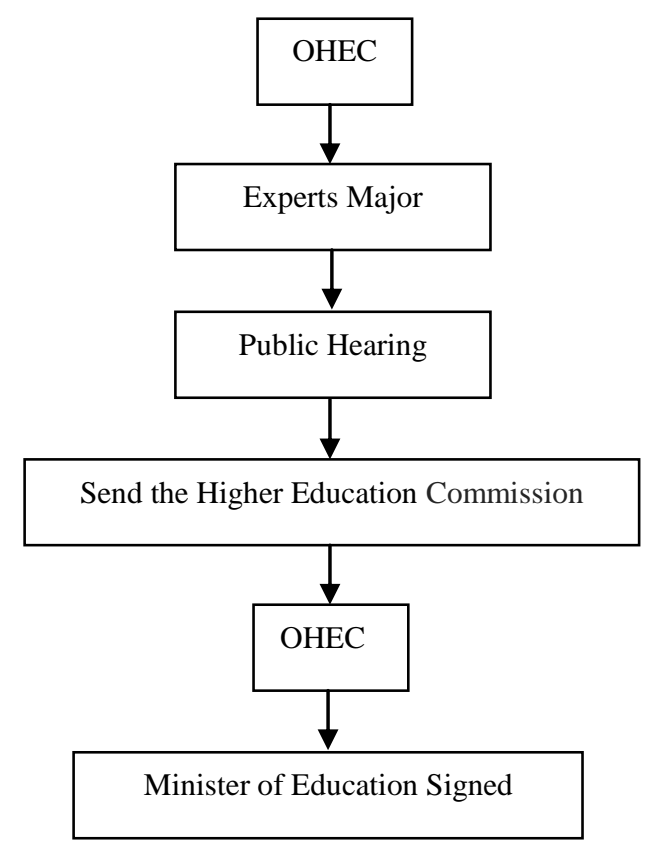

Fig. 1: Procedures implementation of the TQF 1.

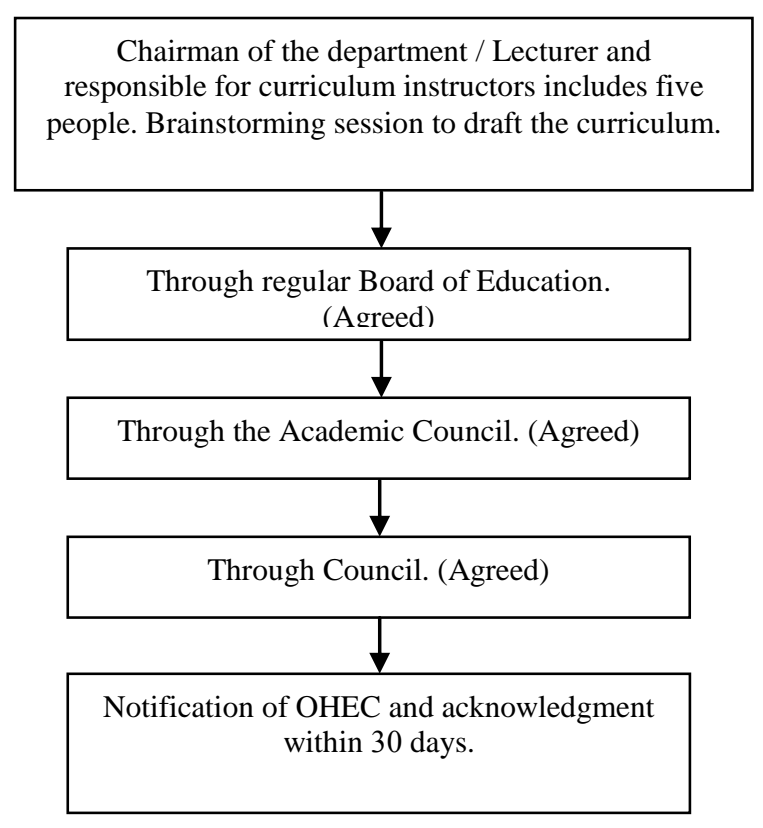

Fig. 2: Procedures implementation of the TQF 2. 
Second, the faculty members that are responsible for curriculum creation retrieve TQF1 from OHEC and create TQF2 based on that. TQF2 is the document that describes the curriculum in details such as curriculum specific information, curriculum management, curriculum structure, curriculum outcome, a list of all instructors, evaluation of curriculum, etc. After designing the curriculum in detail, the TQF2 is submitted to Board of Education, Academic Council, Council, and OHEC accordingly as shown in "Fig. 2,"

Third, based on TQF2, TQF3 and TQF4 are the documents that give each subject in a curriculum in details such as topics, schedules, teaching methods, evaluation methods, etc. This information depends on each instructor, which would be changed every semester. The difference between TQF3 and TQF4 is that TQF3 is the document for an in-class subject such as mathematics and TQF4 is the document for an on-thefield subject such as an internship. Both documents are created in a very similar process as shown in "Fig. 3 and Fig. 4," The process starts by the lecturers read the subject information from TQF2 and writes the TQF3 or TQF4 documents. Then the lecturers submit those documents to the instructors that are responsible for the curriculum before the semester starts.

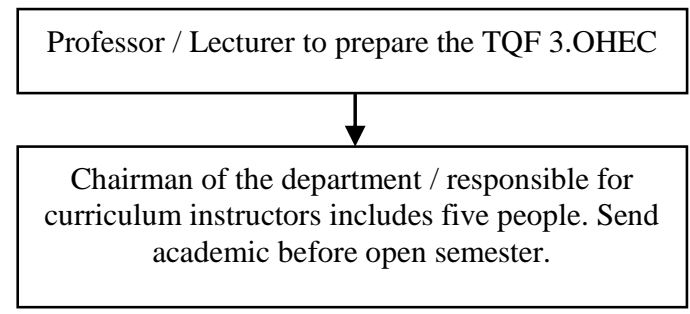

Fig. 3: Procedures implementation of the TQF 3.

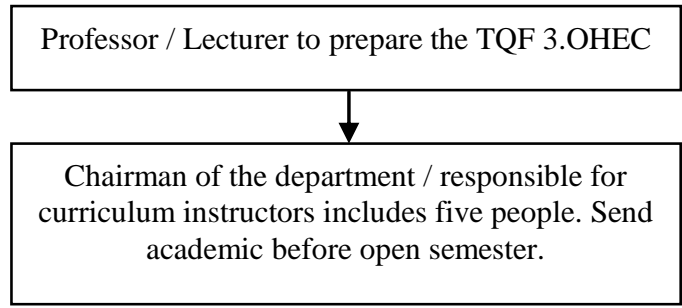

Fig. 4: Procedures implementation of the TQF 4.

Fourth, once the class has been taught, each lecturer creates TQF5 and TQF6 documents at the end of the semester. In TQF5 and TQF6 documents, the lecturers mention the problems and the suggestions found during the classes in the semester. Then the lecturers submit these documents to the instructors that are responsible for the curriculum, as shown in "Fig. 5 and Fig. 6," The difference between TQF5 and TQF6 is the same and the difference between TQF3 and TQF4. These problems and suggestions will be used by the instructors that will be teaching the same subject in the next semester to adjust the teaching methods and the learning methods.

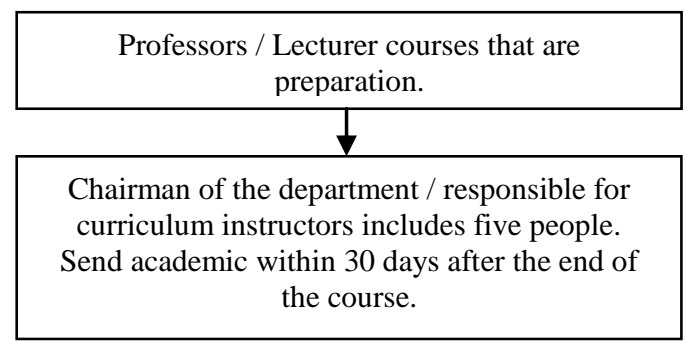

Fig. 5: Procedures implementation of the TQF 5.

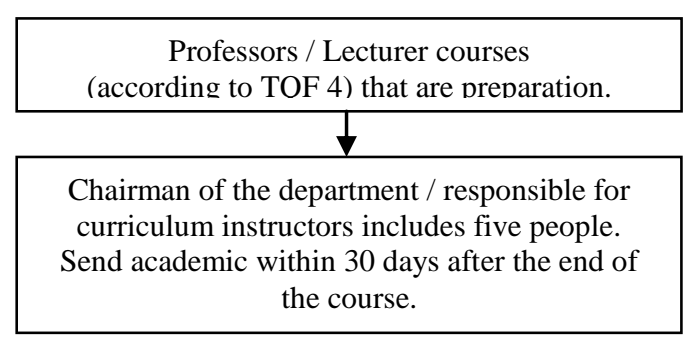

Fig. 6: Procedures implementation of the TQF 6.

Finally, the TQF7 is the document that reports the outcomes of the curriculum. It is an annual report that gathers all the information from TQF3 to TQF6 and summarizes the operation during each year. The TQF7 documents of each year are used as the basic information when modifying the curriculum after using for 5 years. Then the whole process will start again from TQF1 to TQF7. As the result, the curriculum gets better 
quality from the problems and suggestions from the past. "Fig. 2," shows the relationship of all the documents and the related committees that involve in the process.

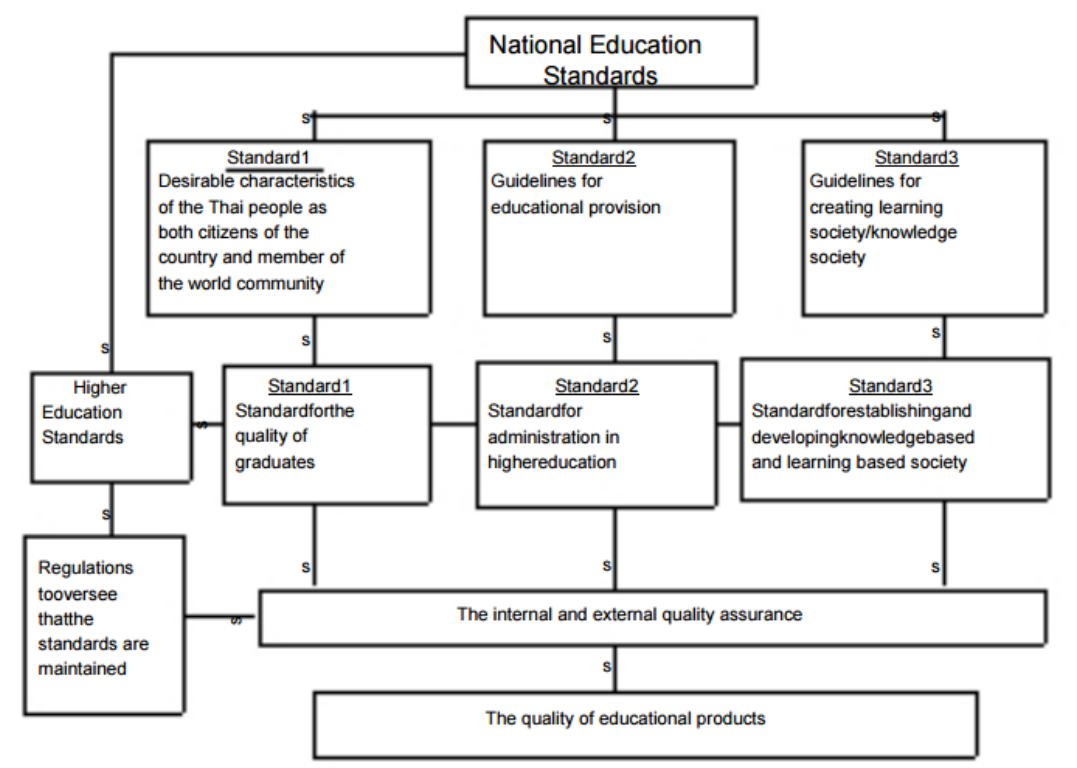

Fig. 7: The relationship between education standards and quality assurance.

\subsection{Existing studies}

Traditionally, government official documents are papers as they are the proof of evidence in a transaction. Storing paper-based documents have several retrieval limitations, which are information processing and loss of document. Fujiwara [3] proposed the idea of document management system. The document management system handles document management, document storage format, document repository, and discernment information. The discernment information table includes the document information and the user information. Hajmiragha [4] proposed the remote access of predesigned user in the document management system, which allows secure document collaboration among users. Il [5] proposed the system allows the image to be searchable. First, the image input unit in the document management system that supports electronic image as an input. Then the text extraction unit performs text recognition on the image. Finally, the text information is sent to the indexer. Zhuge [6] proposed the activity document framework, which allows documents to be self-representable, self-explainable, and self-executable. The document is represented in 4 areas which are granularity hierarchy, template hierarchy, background knowledge, and the semantic link between fragments. As the result, the system improves the efficiency of information retrieval, the preciseness, mobility of information services, and intelligent information services. Armenska [7] compared information retrieval models for question answering. The discussion in [7] focuses on which information retrieval model suits which type of samples. Wybrow [8] extended the system by proposing a new adaptive approach to reading the document. The system dynamically generates the navigation to another document for the links or the references. Heinicke et al. [9] discussed the functionality needed to improve the usability of the existing document management system by proposing a new document representation that is interactive and gets the overview of the document structure. In [9], the result showed that using a zoomable treemap is the most appropriate visualization type for the data in document management system and is a recommend interactive presentation for the data structure. Marujo et al. [10] proposed an approach to summarize multiple documents in document management system. In [10], the proposed method adapts the text extraction process in a single document, uses the proposed model to construct relevant document linkage, and performs multiple documents summarization again. Kao and Liu [11] introduced a new design of the document management system in a private cloud environment. Its concept supports information availability and accessibility from any places.

We have Developed the M-TQF to modify the operation from a manual systems to an information systems. With all the existing studies, our proposed model called M-TQF was designed to support 
curriculum database management in Information Technology field that complies with TQF standard and specifically for the Rajamangala University of Technology Suvarnabhumi.

\section{Methodology}

Our methodology is based on modified vector space model for our samples, as it is the most suitable model mention in [7]. Then we improved the model and proposed the M-TQF model in our work. The MTQF can store the data subjects, data instructors, group classes, courses in TQF documents, schedules and other information. Every table has a relationship and links information on the display as required.

According to the TQF requirements, there are seven types of the document named as TQF1 to TQF7. We take the current document workflow and redesign the process by attaching M-TQF system to reduce redundancy of the tasks. In the first step, all the lecturers need to submit TQF3 and TQF4 documents before starting each semester by 30 days. Then the documents are reviewed by the responsible instructors and the staffs. Finally, the documents are published to the outsiders upon the approval of the complete document as shown in "Fig. 8,"

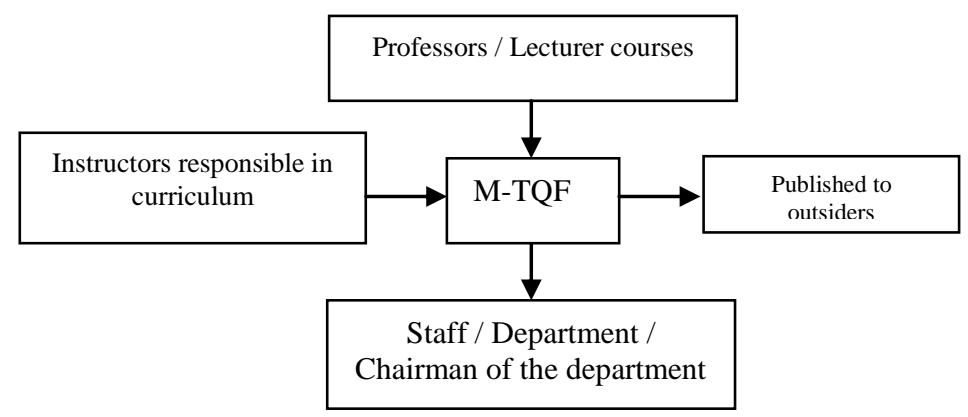

Fig. 8: Sequence processing TQF 3 and 4 on M-TQF.



Fig. 9: Sequence processing TQF 5 and 6 on M-TQF.

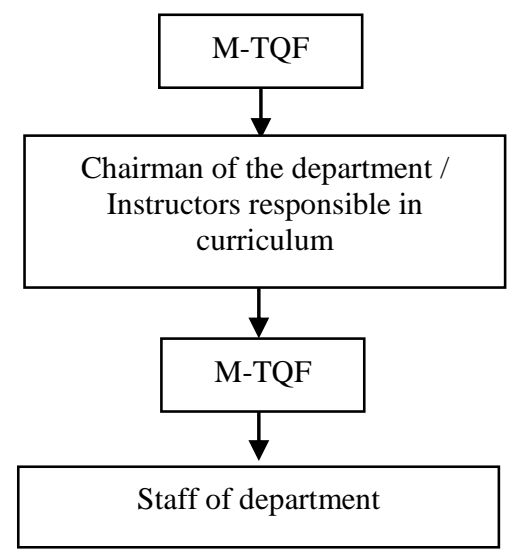

Fig. 10: Sequence processing TQF 7 on M-TQF. 
Once the class has been taught, the instructors make the TQF5 and TQF6 documents and upload to MTQF within 30 days of the last class. Then the documents are reviewed by the lecturer curriculum and the staffs as shown in "Fig. 9," Once M-TQF has collected all the TQF3, TQF4,TQF5, and TQF6 documents. MTQF automatically process all the related documents in that semester and generated the final document called TQF7 as shown in "Fig. 10,"

\section{Evaluation of Result}

In order to evaluate the system, we ask five experts in Information Technology field to evaluate using questionnaires and use the assessment technique of the curriculum in [12]. Three main aspects are measured in the system. These are the system design, the user interface, and the security. The results of the evaluation are shown in Table 2. According to the result, all the aspects of the measurement pass the very good status, which has average, not less than 4.00. The system also achieves the ease of sending TQF document and checking TQF documents, which are the main core of the system.

Table 2. Evaluation Result of Interactions Designed by Experts

\begin{tabular}{|c|l|c|c|c|}
\hline \multicolumn{1}{|c|}{ Nopics } & \multicolumn{1}{|c|}{$\begin{array}{c}\text { Point } \\
\text { Average }\end{array}$} & S.D. & $\begin{array}{c}\text { Meaning of } \\
\text { Satisfaction }\end{array}$ \\
\hline \multicolumn{1}{|c|}{ The System Design } & 4.00 & 0.65 & Very good \\
\hline 1 & Ease of used system & 4.00 & 0.71 & Very good \\
\hline 2 & Appropriate color & 4.20 & 0.45 & Very good \\
\hline 3 & Clarity of text on the screen & 3.80 & 0.84 & Very good \\
\hline 4 & Clarity of the picture & 4.40 & 0.55 & Excellent \\
\hline 5 & The suitability of interaction & 3.60 & 0.55 & Very good \\
\hline The User Interface & 4.36 & 0.57 & Excellent \\
\hline 1 & Ease of use & 4.20 & 0.45 & Very good \\
\hline 2 & ease of sending TQF & 4.60 & 0.55 & Excellent \\
\hline 3 & Ease of check TQF & 4.40 & 0.55 & Excellent \\
\hline 4 & Appropriate for Reporting & 4.20 & 0.84 & Very good \\
\hline 5 & Ease of exit & 4.40 & 0.55 & Excellent \\
\hline The Security & 4.10 & 0.64 & Very good \\
\hline 1 & An overview of the right to security of TQF & 3.80 & 0.84 & Very good \\
\hline 2 & The appropriateness of the permissions for various levels & 4.60 & 0.55 & Excellent \\
\hline 3 & The suitability of the security administrator & 4.20 & 0.45 & Very good \\
\hline 4 & The accuracy of the system within the security system & 3.80 & 0.45 & Very good \\
\hline \multicolumn{2}{|l|}{ Total average } & 4.16 & 0.63 & Very good \\
\hline
\end{tabular}

\section{Conclusion and Future Work}

In this work, we define the current problems in Thailand Qualification Framework that all the universities in Thailand must be using. Then, to reduce the redundancy and complexity of the framework, the M-TQF was proposed to simplify the process. After that, the system was evaluated by the experts. The results showed that the proposed system passes the high level of standard. Furthermore, we continue to improve the system especially the design of the user interface and automate the tasks of generating the new TQF3 and TQF4 for the next semester.

\section{Future Plans}

We continue to develop M-TQF comes to the design, the performance under TQF, sequence analysis, processing, storage system, and process monitoring, questionnaires, Evaluation of interactions designed by experts. Researchers were operating continuously. By programming the system to submit to a test user, improve satisfaction of users. The research operation summaries and published the next step. 


\section{Acknowledgment}

This work cannot complete without the great support from all the faculty members and the students from Faculty of Science and Technology in Suphanburi campus of the Rajamangala University of Technology Suvarnabhumi for taking the survey and give very useful suggestions for the development of the system.

\section{References}

[1] Office of the higher education commission. (December 2015). Thailand qualification framework for higher education. [Online]. Available: www.mua.go.th.

[2] Misistry of education commission on higher education bureau of standards and evaluation. (December 2015). Mannual for the internal quality assurance for higher education institutions. [Online]. Available: www.mua.go.th.

[3] Fujiwara, A. (2006). Document management system. U.S. Patent No. 7,047,492. Washington, DC: U.S. Patent and Trademark Office.

[4] Hajmiragha, M. (2001). Document management system. U.S. Patent No. 6,289,460. Washington, DC: U.S. Patent and Trademark Office.

[5] Il, Y. (2010). U.S. Patent No. 7,668,814. Washington, DC: U.S. Patent and Trademark Office. Hai Zhuge. (October 2003). Active e-document framework ADF: model and tool. Information \& Management, sciencedirect. [Online]. 14(1). pp. 87-97.

[6] Zhuge, H. (2003). Active e-document framework ADF: model and tool.Information \& management, 41(1), 87-97.

[7] Armenska, J., \& Zdravkova, K. (2012, September). Comparison of information retrieval models for question answering. In Proceedings of the Fifth Balkan Conference in Informatics (pp. 162-167). ACM.

[8] Wybrow, M. (2013, September). Reimagining digital publishing for technical documents. In Proceedings of the 2013 ACM symposium on Document engineering (pp. 285-286). ACM.

[9] Heinicke, A., Liao, C., Walbaum, K., Bützler, J., \& Schlick, C. M. (2015). User centered evaluation of interactive data visualization forms for document management systems. Procedia Manufacturing, 3, 5427-5434.

[10] Marujo, L., Ling, W., Ribeiro, R., Gershman, A., Carbonell, J., de Matos, D. M., \& Neto, J. P. (2016). Exploring events and distributed representations of text in multi-document summarization. Knowledge-Based Systems, 94, $33-42$.

[11] Kao, C. H., \& Liu, S. T. (2013). Development of a Document Management System for Private Cloud Environment. Procedia-Social and Behavioral Sciences, 73, 424-429.

[12] Tiantong, M., Design and development of Computer Assisted Instruction., Bangkok, Thailand: King Mongkut's Institute of Technology North Bangkok, 2015, ch. 8, pp. 207-234. 